\title{
THE SHIFT AND SHAPE OF SPECTRAL LINES
}

\author{
BY \\ ROBERT G. B BRENE, Jr.
}

\author{
PERGAMON PRESS \\ NEW YORK - OXFORD - LONDON - PARIS \\ 1961
}

